NHS

Greater Glasgow and Clyde

\title{
Data analysis of a pilot self completed questionnaire for patients attending a men-having-sex-with-men (MSM) clinic
}

\author{
Philip Bayman (Medical Student), Dr Rebecca Gilson. Steve Retson Project, Sandyford Central
}

\section{$\underline{\text { Introduction }}$}

- Data from Health Protection Scotland has shown an increase in syphilis ${ }^{1}$ and rectal gonorrhoea ${ }^{2}$ diagnoses which are STIs that mostly impact the MSM population .

-Increasingly there is a need for MSM services to provide more efficient care, appropriately allocate resources and see high risk patients in a timely manner.

-Anecdotal evidence suggested that the majority of MSM currently attending services were low risk.

-The questionnaire was created to discover the characteristics and risk factors of MSM attending dedicated services.

-The aim of the questionnaire was to assess the reasons for attending in order to focus the skill mix of the clinic and improve patient pathways.

\section{Methods}

-Anonymous self completed questionnaire

-Questions on risk factors for sexually transmitted infections, bloodborne virus transmission, usage of post-exposure prophylaxis and preexposure prophylaxis.

-28 April - 11 July, 2016.

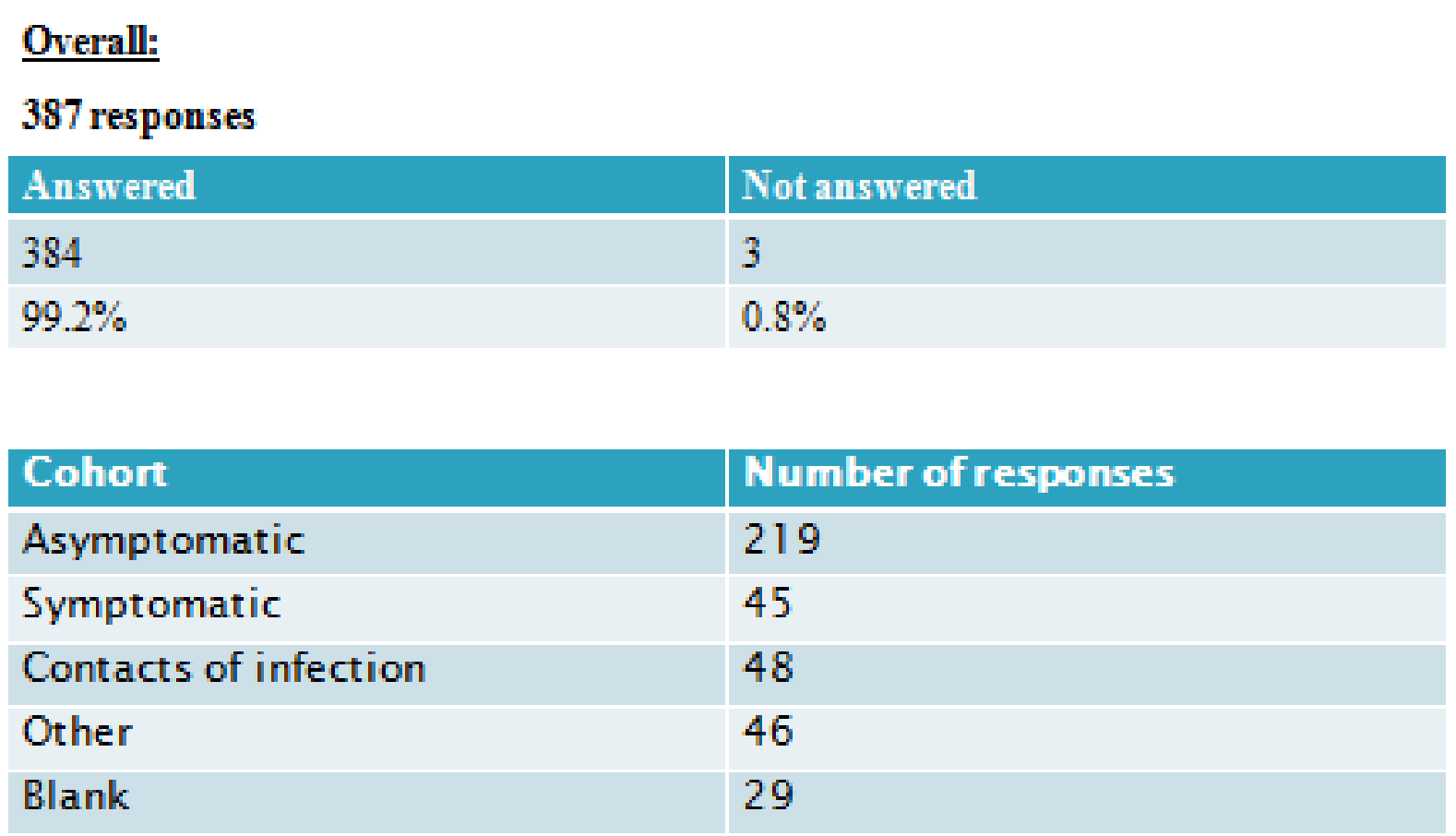

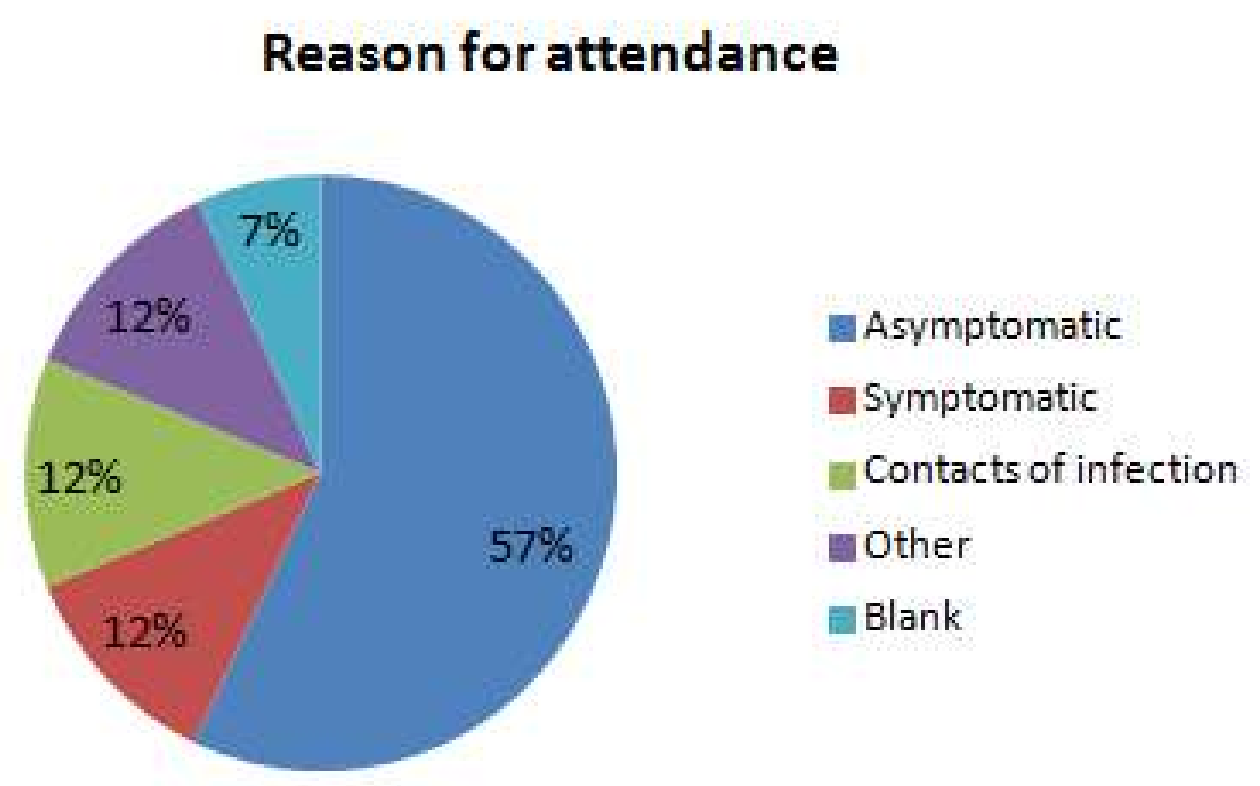

\section{$\underline{\text { References }}$}

1. Health Protection Scotland, HPS Weekly Report - Syphilis in Scotland 2015: Update (PDF), (Glasgow: HPS, 2016)

2. Health Protection Scotland, Gonococcal antibiotic surveillance in Scotland (GASS): prevalence, patterns and trends in 2015 (PDF), (Glasgow: HPS, 2016) $\underline{\text { Results }}$

-Acceptability of the questionnaire (based on response rate) was $99.2 \%$.

-For contacts of infection, HIV and gonorrhoea were the most prevalent.

- $15 \%$ of clients did not use condoms.

-49\% of clients did not know a sexual partner's HIV status.

-31 users of PEP, 5 users of PrEP.

-83\% of clients have had anal sex in the past 3 months.

-4.3 partners was the average for sexual partners in the past 3 months.

-15\% of clients had engaged in group sex during the past 3 months.

-2\% of clients had paid for sex.

$.5 \%$ of clients were HIV positive.

.7\% of clients had used chemsex drugs (MCAT, GBL, methamphetamine)

-3\% of clients were never having sober sex.

-4\% of clients felt their drug and alcohol use had a negative impact.

\section{Analysis of symptomatic patients}

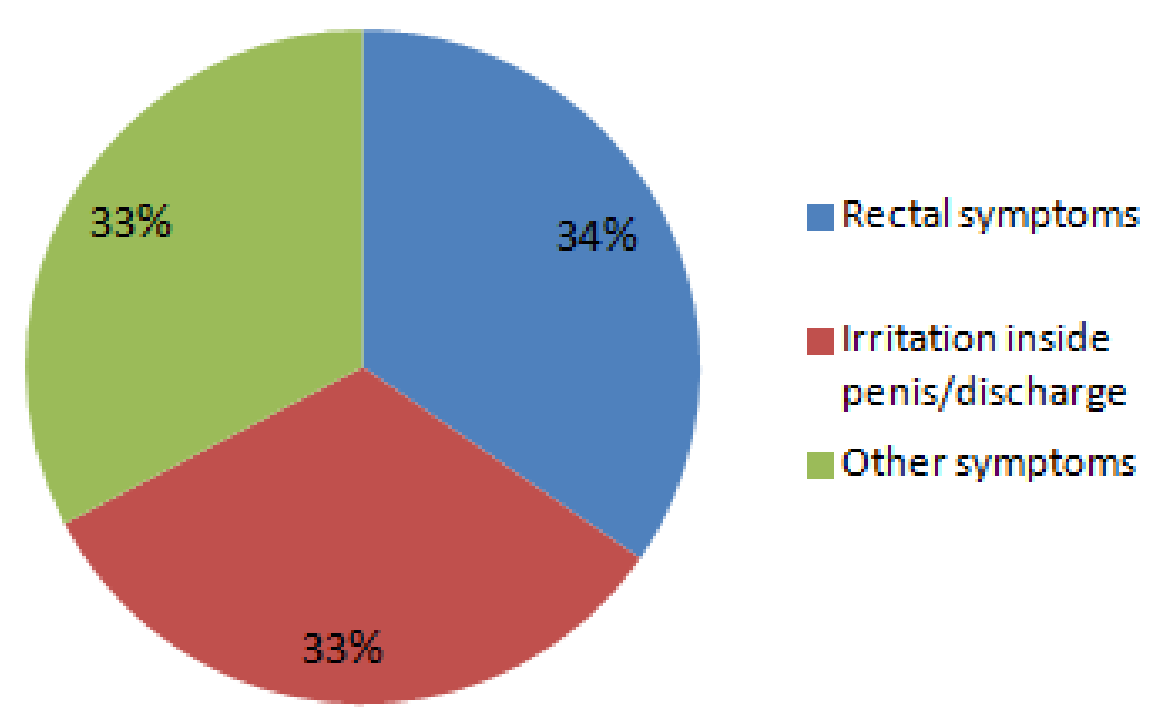

Patients attending due to contact with infection

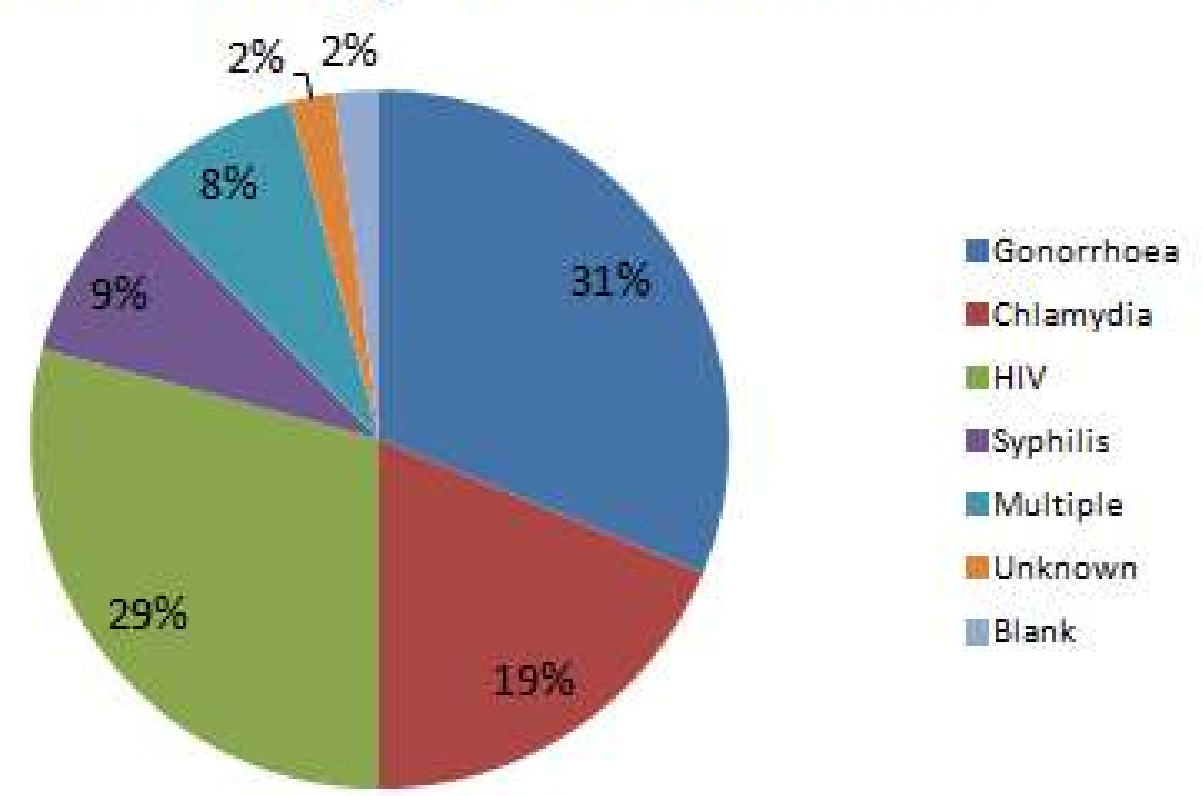

Discussion

-The study showed a majority low-risk, asymptomatic MSM population using the dedicated clinic (57\%).

-The survey has influenced clinic redesign with the introduction of several test-only clinics for the low risk cohort.

-There are plans to stream attendees more efficiently to see a Health Advisor, Clinician or Health Care Assistant.

-This pilot questionnaire will form the basis for online self taken histories in the future.

However the clinic may not be seeing the high-risk patients who would benefit from senior medical input rather than just a sexual health screen. 